\title{
Information Technology and Behavior: An Overview of a Symbiotic Relationship
}

The developed nations are presently going through a revolutionary period the likes of which have probably not been experienced for well over a century. It is a revolution that affects all parts of the nations: individuals, groups, cultures, and societies. It affects people's social, leisure, and working life-even their education and health care. The present-day information revolution is proving as beneficial and as traumatic as the industrial revolution during the nineteenth century. Computers are showing themselves to be as great a force for changing the face of society as steam in its day.

There is no doubt that the benefits that information technology brings to society are numerous and are becoming more apparent with each passing month. Advances in the hardware and software that constitute such technology are so rapid that new developments appear almost daily: newspapers can be written, edited, composited, and even substantially reworked quickly, easily, and cheaply. Individuals working at home can communicate with other individuals at other homes-not simply sending words through the telephone, but also passing. very complex material through computers linked to each other with only a telephone line (which will eventually be replaced by a satellite). Computers are used to help teachers teach, doctors practice medicine, and designers create innovative structures. In Europe, French telephone subscribers are provided with free computer terminals through which they can order goods and services and carry out many tasks of everyday living from the living room. All of these advances, and many more whose examples are provided in the articles in this special issue, have occurred over the past few years. The abundance of examples illustrates the massive impact that such systems have already had on normal living, working, and leisure. Certainly, it does not take a quantum leap in thinking to visualize their likely impact in only a few years' time.

To ensure that developed societies maintain their lead in this new technology, most industrialized countries have instituted detailed research programs with the aim of developing further knowledge about information - its creation, manipulation, and transmission. The Alvey Programme in the United Kingdom, and the European ESPRIT program are just two examples of directed research programs that over the globe have consumed many millions of pounds, dollars, ECUs, yen, marks, francs, and so on. There is no doubt that such programs have spurred information technology manufacturers and developers on to greater heights.

Despite such positive statements and predictions, however, information technology is still used only by a small proportion of the people who could benefit from it. Even those who use it often do not do so to its fullest extent. In the literature, examples abound of organizations that apparently have wasted money on installing systems that have remained idle, or of individuals or groups who have reacted nega-

Current Psychological Research \& Reviews, Summer 1986, 91-93. 
tively toward the systems despite their potential benefits. With such examples as evidence, then, it would appear that society's financial investments may not be as good as previously thought, that there is a mismatch between society's expectations of the benefits of new technology and the actual results obtained. As time goes on, with the mismatch and gap growing, it becomes increasingly imperative to determine why the problems exist. Money and opportunities are being lost at an alarming rate.

The answers to such problems are both simple and complex: an apparently simple, and perhaps obvious, response leads to a multitude of further, more complex, questions-questions that are only beginning to be answered. The purpose of this special issue is to illustrate some of these questions and their answers.

The simple, embracing answer is that despite the major (engineering) advances made in the field of information technology, one important link in the operating chain is frequently forgotten-people. It is people who have to operate the machines developed, who have to interact with them to make them function as they wish. It is people who have to accept the systems and their intrusion into work and home. It is people who are often called on to adapt their habits to the dictates of the system and who frequently cannot do so. The simple answer, then, is that if people do not, or will not, or cannot, do these things then the system will not function adequately-and sometimes not at all. In short, the application of psychology and of behavioral information and models to the new technology is often insufficient or missing.

From this standpoint it could be argued that, far from psychology's being conceived as being some small adjunct to information technology design, the real truth is that psychology has so much to offer the new technology that it is indispensibleeven that information technology is in some way an adjunct to psychology! Until the time comes (possibly in the not-too-distant future) when machines can design and operate other machines-without any human input at all at any stage-there is unlikely to be any aspect of information technology design, application, or implementation that does not require the skills, knowledge, and models of many branches of behavioral study. Again, the articles within this special issue will illustrate, in their own areas, the truth of this proposition.

Underlying the simple answer, however, are many complex behavioral issues concerning attitudes, skills, cognition, and so on, all of which have important implications for the successful implementation and utilization of information technology systems. Psychologists recognize that answers to questions posed in these areas are neither simple nor obvious. It must be even more the case, therefore, that psychology is an indispensible discipline as far as information technology is concerned.

However, psychology should not become smug about this state of affairs. Unfortunately, many of the questions being posed by information technology designers are not being answered fully by psychologists-either because the answers are not available or because the questions are falling on deaf ears. Furthermore, whereas it is undoubtedly the case that modern information technology cannot progress adequately without behavioral input, it is also the case that psychological knowledge and theories have had to be extended, refined, or even abandoned because of the data arising as a result of involvement in the information technology field. As Kennedy 
shows in this issue, for example, our conception of the processes involved in reading and eye movements has changed as a result of studies that have considered the design and arrangement of text on computer screens.

Also in this special issue, Rogers demonstrates the symbiotic relationship between our understanding of the cognitive processes controlling recognition and retention of iconic and graphic images and the need to design appropriate stimuli for the information technology user. Shaffer considers the development of models of typing skills as a result of the new technology, which allows much faster and more easily corrected input. Berry and Broadbent's studies illustrate how our understanding of cognitive and decision-making processes need to be refined in the light of artificial intelligence research. Kahn and Cooper consider the stresses that modern-day computing places on individuals and the ways in which understanding and documenting these stresses have been of value in formulating more general stress models. Waterworth and Holmes, and Ferrari as well, consider the relationship between our normal communication models-of speech and dialogue-and their refinement as a result of new systems and interfaces.

On the applications side of the area, too, information technology has forced psychologists to reexamine the basis of their theories. D'Arcy considers how computerassisted learning and education has resulted in changes in teaching practice and theory. Clegg and Corbett perform the same valuable service for computer-aided manufacturing.

The relationship between psychology and information technology, then, is not one of master and servant but one of equal partners. Each needs the other to progress beyond the stage in which they presently find themselves. There is no doubt, for example, that designers of information technology systems could continue to exist without significant behavioral input; they have done so until the present. Equally, there is no doubt that psychology will survive in the absence of information technology; its multifaceted nature provides the attraction to many thousands of psychologists around the world. However, there is also no doubt that to progress and to refine what in both cases are relatively crude models of information or human behavior, each needs the other in what is very much a symbiotic relationship. Taking only nine different areas of the two fields, the articles within this special issue illustrate and emphasize this point well.

David J. Oborne

Guest Editor,

Department of Psychology,

University College of Swansea 